\title{
CENTROAFFINE IMMERSIONS OF CODIMENSION TWO AND PROJECTIVE HYPERSURFACE THEORY
}

\author{
KATSUMI NOMIZU* AND TAKESHI SASAKI**
}

Affine differential geometry developed by Blaschke and his school [B] has been reorganized in the last several years as geometry of affine immersions. An immersion $f$ of an $n$-dimensional manifold $M$ with an affine connection $\nabla$ into an $(n+1)$-dimensional manifold $\tilde{M}$ with an affine connection $\tilde{\nabla}$ is called an affine immersion if there is a transversal vector field $\xi$ such that $\tilde{\nabla}_{X} f_{*}(Y)=$ $f_{*}\left(\nabla_{X} Y\right)+h(X, Y) \xi$ holds for any vector fields $X, Y$ on $M^{n}$. When $f: M^{n} \rightarrow$ $\mathbf{R}^{n+1}$ is a nondegenerate hypersurface, there is a uniquely determined transversal vector field $\xi$, called the affine normal field, an essential starting point in classical affine differential geometry. The new point of view allows us to relax the nondegeneracy condition and gives us more freedom in choosing $\xi$; what this new viewpoint can accomplish in relating affine differential geometry to Riemannian geometry and projective differential geometry can be seen from [NP1], [NP2], [NS] and others. For the definitions and basic formulas on affine immersions, centroaffine immersions, conormal (or dual) maps, projective flatness, etc., the reader is referred to [NP1]. These notions will be generalized to codimension 2 in this paper.

In this paper we present a systematic study of centroaffine immersions of an $n$-manifold into $\mathbf{R}^{n+2}-\{0\}$. Such immersions were studied in [W] by adhering to the original features (including apolarity and local convexity assumption) of the Blaschke theory as much as possible. Our approach is more general in that we follow the spirit of the recent development mentioned above. In particular, our work is motivated by, and applied to, projective differential geometry.

The paper is organized as follows. In Section 1 we develop the basic machinery for centroaffine immersions of codimension 2, obtain two fundamental forms $h$ and $T$ and two cubic forms $C$ and $\delta$. The vanishing of $T$ or $h$ is given a geometric

Received November 15, 1991.

* The work of the first author is supported by an Alexander von Humbold research award at Technische Universität Berlin and Max-Planck-Institut für Mathematik, Bonn.

** The work of the second author is supported by Max-Planck-Institut für Mathematik, Bonn. 
interpretation (Propositions 1.3, 1.4, 1.5). In Section 2, we consider lifts $f: M \rightarrow$ $\mathbf{R}^{n+2}-\{0\}$ of a given immersion $F: M \rightarrow \mathbf{P}^{n+1}$ and find projective invariants through such affine models $f$. In Section 3, we define the dual mapping of $F: M \rightarrow$ $\mathbf{P}^{n+1}$ by means of a lift $f$ and prove a result about selfdual immersions (Proposition 3.5). In Section 4, we study projective flatness and umbilicity for $f: M \rightarrow \mathbf{R}^{n+2}-$ \{0\} (Theorem 4.1) and its projective interpretation (Theorem 4.3). In Section 5, we prove a number of uniqueness theorems for centroaffine immersions $M \rightarrow \mathbf{R}^{n+2}-$ $\{0\}$ as well as for immersions $M \rightarrow \mathbf{P}^{n+1}$, among which Theorems 5.7, 5.8 and 5.9 are the main results. In Section 6 , we show that $f: M \rightarrow \mathbf{R}^{n+2}-\{0\}$ for which $\nabla h=0$ and rank $h \geq 2$ lies on a quadratic cone (Theorem 6.3)-a generalization of the classical theorem of Pick and Berwald for affine hypersurfaces. In Section 7 , we draw, under the assumption $\nabla T=0$, another geometric conclusion that $M$ lies on a quadratic hypersurface or an affine hyperplane (Theorem 7.3).

\section{§1. Centroaffine geometry of affine submanifolds of codimension two}

We use the following notation throughout this paper. By $D$ we mean the standard flat affine connection of $\mathbf{R}^{n+2}$ and by $\eta$ the radial vector field on $\mathbf{R}^{n+2}-$ $\{0\}: \eta=\sum_{i=1}^{n+2} x^{i} \partial / \partial x^{i}$, where $\left\{x^{1}, \ldots, x^{n+2}\right\}$ is an affine coordinate system. The letter $\omega$ denotes a parallel volume form of $\mathbf{R}^{n+2}$ that is fixed once and for all. Let $M$ be an $n$-manifold and $f$ an immersion of $M$ into $\mathbf{R}^{n+2}-\{0\}$. Assume $f$ is transversal to $\eta$. We choose, at least locally, a vector field $\xi$ along $f$ such that, at each point $x \in M$, the tangent space $T_{f(x)} \mathbf{R}^{n+2}$ is decomposed as the direct sum of the span $\mathbf{R}\{\eta\}$, the tangent space $f_{*} T_{x} M$, and the span $\mathbf{R}\{\xi\}$. According to this decomposition, the vectors $D_{X} \eta, D_{X} f_{*} Y$, and $D_{X} \xi$, where $X, Y$ are vector fields on $M$, have the following expressions:

$$
\begin{aligned}
& D_{X} \eta=f_{*} X, \\
& D_{X} f_{*} Y=T(X, Y) \eta+f_{*}\left(\nabla_{X} Y\right)+h(X, Y) \xi, \\
& D_{X} \xi=\rho(X) \eta-f_{*}(S X)+\tau(X) \xi .
\end{aligned}
$$

An $n$-form $\theta$ is defined by

$$
\theta\left(X_{1}, \ldots, X_{n}\right)=\omega\left(f_{*} X_{1}, \ldots, f_{*} X_{n}, \xi, \eta\right) .
$$

Thus we have several objects associated with $\xi$. They have the following properties.

PROPOSITION 1.1.

(1) $\nabla$ is a torsion-free affine connection on $M$. 
(2) $h$ and $T$ are symmetric tensors.

(3) $\nabla_{X} \theta=\tau(X) \theta$.

In the following, we occasionally identify a tangent vector (field) $X$ with its image $f_{*} X$ if there is no danger of confusion. Let $R^{D}$ and $R$ denote the curvature tensors of the connection $D$ and the connection $\nabla$, respectively. Using (1.1), we get

$$
\begin{aligned}
D_{X} D_{Y} Z= & D_{X}\left(T(Y, Z) \eta+\nabla_{Y} Z+h(Y, Z) \xi\right) \\
= & X(T(Y, Z)) \eta+T(Y, Z) X \\
& +T\left(X, \nabla_{Y} Z\right) \eta+\nabla_{X} \nabla_{Y} Z+h\left(X, \nabla_{Y} Z\right) \xi \\
& +X(h(X, Z)) \xi+h(Y, Z)(\rho(X) \eta-S X+\tau(X) \xi)
\end{aligned}
$$

and

$$
D_{[X, Y]} Z=T([X, Y], Z) \eta+\nabla_{[X, Y]} Z+h([X, Y], Z) \xi .
$$

Hence we get

$$
\begin{aligned}
R^{D}(X, Y) Z= & R(X, Y) Z+T(Y, Z) X-T(X, Z) Y-h(Y, Z) S X+h(X, Z) S Y \\
& +\left\{T\left(X, \nabla_{Y} Z\right)-T\left(Y, \nabla_{X} Z\right)+X(T(Y, Z))-Y(T(X, Z))\right. \\
& -T([X, Y] Z)+\rho(X) h(Y, Z)-\rho(Y) h(X, Z)\} \eta \\
& +\left\{h\left(X, \nabla_{Y} Z\right)-h\left(Y, \nabla_{X} Z\right)+X(h(Y, Z))-Y(h(X, Z))\right. \\
& -h([X, Y] Z)+\tau(X) h(Y, Z)-\tau(Y) h(X, Z)\} \xi \\
= & R(X, Y) Z+T(Y, Z) X-T(X, Z) Y-h(Y, Z) S X+h(X, Z) S Y \\
& +\left\{\left(\nabla_{X} T\right)(Y, Z)-\left(\nabla_{Y} T\right)(X, Z)+\rho(X) h(Y, Z)-\rho(Y) h(X, Z)\right\} \eta \\
& +\left\{\left(\nabla_{X} h\right)(Y, Z)-\left(\nabla_{Y} h\right)(X, Z)+\tau(X) h(Y, Z)-\tau(Y) h(X, Z)\right\} \xi .
\end{aligned}
$$

From the equations

$$
\begin{aligned}
D_{X} D_{Y} \xi= & D_{X}(\rho(Y) \eta-S Y+\tau(Y) \xi) \\
= & X(\rho(Y)) \eta+\rho(Y) X-\left\{T(X, S Y) \eta+\nabla_{X}(S Y)+h(X, S Y) \xi\right\} \\
& +X(\tau(Y)) \xi+\tau(Y)\{\rho(X) \eta-S X+\tau(X) \xi\}, \\
D_{[X, Y]} \xi= & \rho([X, Y]) \eta-S[X, Y]+\tau([X, Y]) \xi,
\end{aligned}
$$

we get

$$
\begin{aligned}
R^{D}(X, Y) \xi= & \rho(Y) X-\rho(X) Y-\nabla_{X}(S Y)+\nabla_{Y}(S X)-\tau(Y) S X+\tau(X) S Y \\
& +S[X, Y]+\{X(\rho(Y))-Y(\rho(X))-T(X, S Y)+T(Y, S X) \\
& \quad+\tau(Y) \rho(X)-\tau(X) \rho(Y)-\rho([X, Y])\} \eta \\
& +\{h(Y, S X)-h(X, S Y)+X(\tau(Y))-Y(\tau(X))-\tau([X, Y])\} \xi \\
= & \rho(Y) X-\rho(X) Y-\left(\nabla_{X} S\right)(Y)+\left(\nabla_{X} S\right)(X)-\tau(Y) S X+\tau(X) S Y \\
& +\left\{\left(\nabla_{X} \rho\right)(Y)-\left(\nabla_{Y} \rho\right)(X)-T(X, S Y)+T(Y, S X)\right. \\
&
\end{aligned}
$$




$$
\begin{aligned}
& +\tau(Y) \rho(X)-\tau(X) \rho(Y)\} \eta \\
& +\left\{h(Y, S X)-h(X, S Y)+\left(\nabla_{X} \tau\right)(Y)-\left(\nabla_{Y} \tau\right)(X)\right\} \xi .
\end{aligned}
$$

Since the connection $D$ is flat, we have equations of Gauss (1.3), of Codazzi (1.4), (1.5), (1.6), and of Ricci (1.7), (1.8):

(1.3) $R(X, Y) Z=h(Y, Z) S X-h(X, Z) S Y-T(Y, Z) X+T(X, Z) Y$

(1.4) $\left(\nabla_{X} T\right)(Y, Z)+\rho(X) h(Y, Z)=\left(\nabla_{Y} T\right)(X, Z)+\rho(Y) h(X, Z)$

(1.5) $\left(\nabla_{X} h\right)(Y, Z)+\tau(X) h(Y, Z)=\left(\nabla_{Y} h\right)(X, Z)+\tau(Y) h(X, Z)$

(1.6) $\left(\nabla_{X} S\right)(Y)-\tau(X) S Y+\rho(X) Y=\left(\nabla_{Y} S\right)(X)-\tau(Y) S X+\rho(Y) X$

(1.7) $T(X, S Y)-T(Y, S X)=\left(\nabla_{X} \rho\right)(Y)-\left(\nabla_{Y} \rho\right)(X)+\tau(Y) \rho(X)-\tau(X) \rho(Y)$

(1.8) $h(X, S Y)-h(Y, S X)=\left(\nabla_{X} \tau\right)(Y)-\left(\nabla_{Y} \tau\right)(X)=d \tau(X, Y)$.

From (1.3) we have

$$
\operatorname{Ric}(Y, Z)=\operatorname{tr} S \cdot h(Y, Z)-h(S Y, Z)-(n-1) T(Y, Z) .
$$

At this point we present the following basic lemma that will be repeatedly used.

Lemma 1.2. Let $V$ be a vector space of finite dimension. Suppose $\sigma$ is a linear form and $h$ a symmetric bilinear form on $V$ such that

$$
\sigma(X) h(Y, Z)=\sigma(Y) h(X, Z) \text { for all } X, Y, Z \in V .
$$

If $\operatorname{rank} h \geq 2$, then $\sigma=0$.

We now study what the vanishing of $T$ and $h$ means for a given immersion $f$ : $M \rightarrow \mathbf{R}^{n+2}-\{0\}$.

PROPOSITION 1.3. If $T$ vanishes and rank $h \geq 2$, then the image of the immersion is contained in an affine hyperplane which does not go through 0 and the vector field $\xi$ is tangent to this hyperplane.

Proof. If $T \equiv 0$, then (1.4) says $\rho(X) h(Y, Z)=\rho(Y) h(X, Z)$. By Lemma 1.2, $\rho=0$ and, therefore, the distribution spanned by $f_{*}\left(T_{x} M\right)$ and $\xi_{x}, x \in M$, is parallel relative to $D$; this implies the result.

The immersion $f$ considered to be a mapping into this hyperplane is an affine immersion of $M$ as a hypersurface, relative to the induced flat connection $D^{\prime}$ on the hyperplane, $f$ satisfies

$$
D_{X}^{\prime} f_{*} Y=f_{*}\left(\nabla_{X} Y\right)+h(X, Y) \xi \text { and } D_{X}^{\prime} \xi=-f_{*}(S X)+\tau(X) \xi .
$$




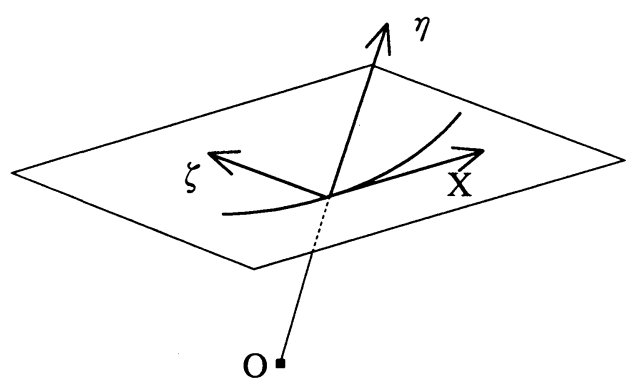

Figure 1 (Prop. 1.3)

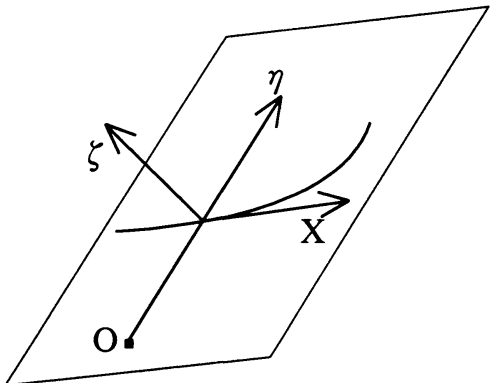

Figure 2 (Prop. 1.4)

PROPOSITION 1.4. If $h$ vanishes and $n \geq 2$, then the image of the immersion is contained in a hyperplane through 0.

Proof. If $h \equiv 0$, then the distribution spanned by $f_{*}\left(T_{x} M\right)$ and $\eta_{f(x)}, x \in M$, is parallel relative to $D$.

In the situation of this proposition, the immersion $f$ defines a centroaffine hypersurface immersion and the tensor $T$ is the fundamental tensor of this immersion.

We put

$$
\begin{aligned}
& C(X, Y, Z)=\left(\nabla_{X} h\right)(Y, Z)+\tau(X) h(Y, Z) \\
& \delta(X, Y, Z)=\left(\nabla_{X} T\right)(Y, Z)+\rho(X) h(Y, Z) .
\end{aligned}
$$

Both are symmetric in their arguments (cf. (1.4), (1.5)). We call $C$ the (first) cubic form and $\delta$ the second cubic form.

We shall next examine how various objects depend on $\xi$. Another choice, say $\xi^{\prime}$, of transversal vector field is related to $\xi$ by

$$
\lambda \xi^{\prime}=\xi+a \eta+f_{*} U,
$$

where $\lambda$ is a nonzero scalar function, $\boldsymbol{a}$ is also a scalar, and $U$ is a tangent vector field. Let $T^{\prime}, \nabla^{\prime}, h^{\prime}, \rho^{\prime}, S^{\prime}$, and $\tau^{\prime}$ denote the quantities corresponding to $\xi^{\prime}$. By (1.1) we have

$$
\begin{aligned}
D_{X} f_{*} Y & =T(X, Y) \eta+f_{*} \nabla_{X} Y+h(X, Y)\left(\lambda \xi^{\prime}-a \eta-f_{*} U\right) \\
& =\{T(X, Y)-a h(X, Y)\} \eta+f_{*}\left(\nabla_{X} Y-h(X, Y) U\right)+\lambda h(X, Y) \xi^{\prime}, \\
D_{X} \xi & =\rho(X) \eta-f_{*} S X+\tau(X)\left(\lambda \xi^{\prime}-a \eta-f_{*} U\right) \\
& =\{\rho(X)-a \tau(X)\} \eta-f_{*}(S X+\tau(X) U)+\lambda \tau(X) \xi^{\prime} .
\end{aligned}
$$


On the other hand, we have

$$
\begin{aligned}
D_{X} \xi= & D_{X}\left(\lambda \xi^{\prime}-a \eta-f_{*} U\right) \\
= & X(\lambda) \xi^{\prime}+\lambda D_{X} \xi^{\prime}-X(a) \eta-a f_{*} X \\
& -\left\{(T(X, U)-a h(X, U)) \eta+f_{*}\left(\nabla_{X} U-h(X, U) U\right)+\lambda h(X, U) \xi^{\prime}\right\} .
\end{aligned}
$$

Term-by-term comparison shows

$$
\begin{aligned}
\nabla_{X}^{\prime} Y & =\nabla_{X} Y-h(X, Y) U \\
T^{\prime}(X, Y) & =T(X, Y)-a h(X, Y) \\
h^{\prime}(X, Y) & =\lambda h(X, Y) \\
\tau^{\prime}(X) & =\tau(X)-X(\log \lambda)+h(X, U) \\
\lambda \rho^{\prime}(X) & =\rho(X)+X(a)+T(X, U)-a h(X, U)-a \tau(X) \\
\lambda S^{\prime} X & =S X+\tau(X) U-a X-\nabla_{X} U+h(X, U) U .
\end{aligned}
$$

Formula (1.13) implies that the conformal class of $h$ is independent of the choice of $\xi$. When the class $h$ is nondegenerate we say that the immersion is nondegenerate. If we assume nondegeneracy, then one can find a vector field $\xi$ so that $\tau=0$ because of (1.14); in this case, $\theta$ is $\nabla$-parallel. We say that this choice of $\xi$ defines (or that the pair $\{f, \xi\}$ is) an equiaffine immersion. Further, we can restrict the choice of $\xi$ so that the form $\theta$ is equal to the volume form of the nondegenerate metric tensor $h$; such $\xi$ is uniquely determined $\bmod \eta$ up to sign. We call this pair $\{f, \xi\}$ a Blaschke immersion of codimension two. Formulas (1.12), (1.13), and (1.16) with $U=0$ show

$$
T^{\prime}(X, Y)+h^{\prime}\left(S^{\prime} X, Y\right)=T(X, Y)+h(S X, Y)-2 a h(X, Y) .
$$

By determining the scalar function $a$ we can assume that $\xi$ is so chosen that

$$
\operatorname{tr}_{h}\{T(X, Y)+h(S X, Y)\}=0 .
$$

If this condition is satisfied, we say that $\xi$ is pre-normalized. In particular, a prenormalized Blaschke immersion $\{f, \xi\}$ is uniquely determined up to sign.

Remark. Consider the situation where $f$ is a nondegenerate hypersurface immersion into an affine hyperplane in $\mathbf{R}^{n+2}-\{0\}$ and where $\underline{\xi}$ is an equiaffine normal relative to this immersion:

$$
D_{X} f_{*} Y=f_{*}\left(\nabla_{X} Y\right)+\underline{h}(X, Y) \underline{\xi} \text { and } D_{X} \underline{\xi}=-f_{*}(\underline{S} X) \text {. }
$$

Then we can regard $f$ as an immersion into $\mathbf{R}^{n+2}-\{0\}$. Let $\xi=\underline{\xi}+a \eta$. Then it is easy to see that $\xi$ is pre-normalized only when $a=\frac{1}{2 n} \operatorname{tr} S$. The associated 
quantities are given by

$$
T(X, Y)=-\frac{1}{2 n} \underline{h}(X, Y), \quad S=\underline{S}-\frac{1}{2 n} I, \quad \text { and } \quad \rho=\frac{1}{2 n} d(\operatorname{tr} \underline{S})
$$

and $\nabla=\underline{\nabla}, h=\underline{h}$, and $\tau=0$.

Let us return to the general situation and prove a result which is more precise than Proposition 1.3.

Proposition 1.5. Assume rank $h \geq 2$. Then the image of the immersion is contained in an affine hyperplane if and only if $T=\alpha h$ for some scalar function $\alpha$.

Proof. Assume $T=\alpha h$. Replace $\xi$ by $\xi^{\prime}=\xi+\alpha \eta$. Then, (1.12) shows that $T^{\prime}$ for $\xi^{\prime}$ vanishes identically. Hence, Proposition 1.3 implies that the image is contained in an affine hyperplane. Conversely, if the image lies in a hyperplane not through 0 , then for a vector field $\xi$ tangent to this hyperplane we get $T=0$. Formula (1.12) also says that the property $T=\alpha h$ is independent of the choice of $\xi$, although the scalar $\alpha$ depends on $\xi$.

We also have

Proposition 1.6. Let $n \geq 3$. Assume $\nabla$ is flat and rank $h \geq 2$. Then the image of the immersion lies on an affine hyperplane and the immersion turns out to be a graph immersion into this hyperplane.

Proof. The condition that $\nabla$ is flat means

$$
R(X, Y) Z=h(Y, Z) S X-h(X, Z) S Y-T(Y, Z) X+T(X, Z) Y=0
$$

Let $\left\{X_{1}, \ldots, X_{r}, X_{r+1}, \ldots, X_{n}\right\}$ be a basis such that $\left\{X_{r+1}, \ldots, X_{n}\right\}$ generates ker $h$ and $h\left(X_{i}, X_{j}\right)=\varepsilon_{i} \delta_{i j}, \varepsilon_{i}= \pm 1$, for $1 \leq i, j \leq r$. For $i \neq j$, choose $k \neq i, j$ $(n \geq 3)$. By letting $X=X_{i}, Y=X_{k}, Z=X_{j}$, (1.18) implies $-T\left(X_{k}, X_{j}\right) X_{i}+$ $T\left(X_{i}, X_{j}\right) X_{k}=0$. Hence, $T\left(X_{i}, X_{j}\right)=0$. For $i \neq j$ let $X=X_{i}, Y=Z=X_{j}$. Then (1.18) implies

$$
h\left(X_{j}, X_{j}\right) S X_{i}-T\left(X_{j}, X_{j}\right) X_{i}=0 .
$$

If $1 \leq j \leq r$, then $S X_{i}=\mu X_{i}$ where $\mu=T\left(X_{j}, X_{j}\right) / h\left(X_{j}, X_{j}\right)$. Since rank $h \geq 2$, this identity holds for all $i$ and $\mu$ is independent of $j$. If $r+1 \leq j \leq n$, then $T\left(X_{j}\right.$, $\left.X_{j}\right)=0$. Therefore, we have

$$
S=\mu I \text { and } T(X, Y)=\mu h(X, Y) .
$$


By changing $\xi$ to $\xi^{\prime}=\xi+\mu \eta$, the equations (1.11), (1.12), and (1.16) allow us to assume $S=0$ and $T=0$. By Proposition 1.3 and Example 3 of [NP1] we get the conclusion.

We conclude this section with the following formulas for later applications.

Proposition 1.7. Under the change of $\xi$ to $\xi^{\prime}=\lambda^{-1}\left(\xi+a \eta+f_{*} U\right)$ the cubic forms $C$ and $\delta$ transform as follows:

$$
\begin{aligned}
\lambda^{-1} C^{\prime}(X, Y, Z)= & C(X, Y, Z)+h(X, Y) h(U, Z)+h(Y, Z) h(U, X) \\
& +h(Z, X) h(U, Y) \\
\delta^{\prime}(X, Y, Z)+\alpha(\lambda)^{-1} C^{\prime}(X, Y, Z) & =\delta(X, Y, Z)+h(X, Y) T(U, Z) \\
& +h(Y, Z) T(U, X)+h(Z, X) T(U, Y)
\end{aligned}
$$

Proof. The first identity is similar to the affine hypersurface case (see [NP2]). The second one is calculated as follows.

$$
\begin{aligned}
&\left(\nabla_{X}^{\prime} T^{\prime}\right)(Y, Z)+\rho^{\prime}(X) h^{\prime}(Y, Z) \\
&= X\left(T^{\prime}(Y, Z)\right)-T^{\prime}\left(\nabla_{X}^{\prime} Y, Z\right)-T^{\prime}\left(Y, \nabla_{X}^{\prime} Z\right)+\lambda \rho^{\prime}(X) h(Y, Z) \\
&= X(T(Y, Z)-a h(Y, Z))-T\left(\nabla_{X}^{\prime} Y, Z\right)+a h\left(\nabla_{X}^{\prime} Y, Z\right) \\
&-T\left(Y, \nabla_{X}^{\prime} Z\right)+a h\left(Y, \nabla_{X}^{\prime} Z\right)+\lambda \rho^{\prime}(X) h(Y, Z) \\
&= X(T(Y, Z))-X(a) h(Y, Z)-a X(h(Y, Z)) \\
&-T\left(\nabla_{X} Y, Z\right)+h(X, Y) T(U, Z)+a h\left(\nabla_{X} Y, Z\right)-a h(U, Z) h(X, Y) \\
&-T\left(Y, \nabla_{X} Z\right)+h(X, Z) T(U, Y)+a h\left(Y, \nabla_{X} Z\right)-a h(U, Y) h(X, Z) \\
&+\{\rho(X)+X(a)+T(X, U)-a h(X, U)-a \tau(X)\} h(Y, Z) \\
&= \delta(X, Y, Z)-a C(X, Y, Z)+h(X, Y)\{T(U, Z)-a h(U, Z)\} \\
&+h(Y, Z)\{T(U, X)-a h(U, X)\}+h(Z, X)\{T(U, Y)-a h(U, Y)\} .
\end{aligned}
$$

\section{§2. Projective hypersurfaces}

Let $\pi: \mathbf{R}^{n+2}-\{0\} \rightarrow \mathbf{P}^{n+1}$ be the natural projection where $\mathbf{P}^{n+1}$ is a projective space of dimension $n+1$. Let $F$ be an immersion of an $n$-manifold $M$ into $\mathbf{P}^{n+1}$. Then, locally, there is an immersion $f$ of $M$ into $\mathbf{R}^{n+2}-\{0\}$ such that $\pi \cdot f$ $=F$. We call $f$ a local lift of $F$ and use the notation $F=[f]$. Another local lift $g$ is written as $g=\phi f$ for some nonzero scalar function $\phi$. In this section we want to obtain ralations of the invariants for $f$ and those for $g$ and, thereby, to find out what invariants can be attached to the immersion $F$.

We first consider the relationship between $f_{*}$ and $g_{*}$. Since

$$
D_{X} g=D_{X}(\phi f)=(X \phi) f+\phi D_{X} f,
$$


we have

$$
g_{*} X=(X \phi) \eta_{f(x)}+\phi f_{*} X=X(\log \phi) \eta_{g(x)}+\phi f_{*} X
$$

where $f_{*} X \in T_{f(x)} \mathbf{R}^{n+2}$ is considered to be in $T_{g(x)} \mathbf{R}^{n+2}$ by parallel translation. For the moment we write $\eta_{g}$ for $\left.\eta\right|_{g(x)}$ and $\eta$ for $\left.\eta\right|_{f(x)}$. Then

$$
g_{*} X=\sigma(X) \eta_{g}+\phi f_{*} X
$$

where $\sigma=d \log \phi$. The quantities for $g$ are denoted with "-". We get the following formulas with respect to the immersions $(f, \xi, \eta)$ and $\left(g, \xi, \eta_{g}\right)$ :

$$
\begin{aligned}
& \bar{\nabla}_{X} Y=\nabla_{X} Y+\sigma(Y) X+\sigma(X) Y \\
& \bar{T}(X, Y)=\operatorname{Hess}_{\log \phi}^{\nabla}(X, Y)-\sigma(X) \sigma(Y)+T(X, Y) \\
& \bar{h}(X, Y)=\phi h(X, Y)
\end{aligned}
$$

where

$$
\operatorname{Hess}_{\log \phi}^{\nabla}=\text { Hessian of } \log \phi=\nabla \sigma .
$$

The proof is straightforward by calculating

$$
\begin{aligned}
D_{X} g_{*} Y= & D_{X}\left\{\sigma(Y) \eta_{g}+\phi f_{*} Y\right\} \\
= & X(\sigma(Y)) \eta_{g}+\sigma(Y) g_{*} X+X(\phi) f_{*} Y+\phi D_{X} f_{*} Y \\
= & X(\sigma(Y)) \eta_{g}+\sigma(Y) g_{*} X+\sigma(X)\left(g_{*} Y-\sigma(Y) \eta_{g}\right) \\
& +\phi\left\{T(X, Y) \eta+f_{*} \nabla_{X} Y+h(X, Y) \xi\right\}
\end{aligned}
$$

which, on the other hand, should be $\bar{T}(X, Y) \eta_{g}+g_{*}\left(\bar{\nabla}_{X} Y\right)+\bar{h}(X, Y) \xi$.

Similarly, by the identity

$$
\begin{aligned}
D_{X} \xi & =\rho(X) \eta-f_{*} S X+\tau(X) \xi \\
& =\bar{\rho}(X) \eta_{g}-g_{*} \bar{S} X+\bar{\tau}(X) \xi,
\end{aligned}
$$

we have

$$
\begin{aligned}
\bar{\tau} & =\tau \\
\phi \bar{\rho}(X) & =\rho(X)+\sigma(S X) \\
\phi \bar{S} & =S .
\end{aligned}
$$

Moreover we can see that

$$
\bar{\theta}=\phi^{n+1} \theta \text {. }
$$

Hence, the conformal class of $h$ is preserved and, if $f$ is equiaffine, i.e. $\tau=0$, then $g$ is also equiaffine relative to the same $\xi$.

Let $\bar{R}$ denote the curvature tensor of $\bar{\nabla}$ and let $\gamma$ and $\bar{\gamma}$ be the normalized 
(i.e., divided by $n-1$ ) Ricci tensors of $\nabla$ and $\bar{\nabla}$, respectively. We see $\bar{R}(X, Y) Z=R(X, Y) Z+\left(\nabla_{X} \sigma\right)(Z) Y-\left(\nabla_{Y} \sigma\right)(Z) X+\sigma(Y) \sigma(Z) X-\sigma(X) \sigma(Z) Y$ and

$$
\bar{\gamma}(X, Y)=\gamma(X, Y)-\left\{\left(\nabla_{X} \sigma\right)(Y)-\sigma(X) \sigma(Y)\right\}
$$

Hence we get

$$
\bar{T}(X, Y)+\bar{\gamma}(X, Y)=T(X, Y)+\gamma(X, Y) .
$$

Assume that $h$ is nondegenerate and that $\{f, \xi\}$ is a pre-normalized Blaschke immersion. Then the immersion $g$ has a similar normalization. Let $\tilde{\xi}$ be an associated vector field which can be written as

$$
\lambda \tilde{\xi}=\xi+a \eta_{g}+g_{*} U .
$$

By computation, we see that the identities

$$
\lambda=\phi, \quad \phi h(U, X)=\sigma(X), \quad 2 a+\sigma(U)=0
$$

determine $\tilde{\xi}$. Let $(\tilde{\nabla}, \tilde{h}, \tilde{S}, \tilde{T}, \tilde{\rho})$ be the data for $\tilde{\xi}$. They are given by the following formulas:

$$
\begin{aligned}
\tilde{\nabla}_{X} Y= & \nabla_{X} Y+\sigma(X) Y+\sigma(Y) X-\phi h(X, Y) U \\
\tilde{h}(X, Y)= & \phi^{2} h(X, Y) \\
\tilde{T}(X, Y)= & T(X, Y)+\left(\nabla_{X} \sigma\right)(Y)-\sigma(X) \sigma(Y)-a \phi h(X, Y) \\
\phi^{2} \tilde{S} X= & S X-a \phi X-\phi\left\{\nabla_{X} U+\sigma(X) U+\sigma(U) X\right\}+\phi^{2} h(U, X) U \\
\phi^{2} \tilde{\rho}(X)= & \rho(X)+\sigma(S X)+\phi X(a)-a \phi^{2} h(U, X) \\
& +\phi\left\{\left(\nabla_{X} \sigma\right) U-\sigma(X) \sigma(U)+T(U, X)\right\}
\end{aligned}
$$

We define a quadratic form $\mathscr{T}$ by

$$
\mathscr{T}(X, Y)=T(X, Y)+h(S X, Y) .
$$

Then we can prove easily the following formulas:

Proposition 2.1.

(1) $\tilde{C}=\phi^{2} C$.

(2) $\tilde{\mathscr{T}}(X, Y)=\mathscr{T}(X, Y)+\phi C(U, X, Y)$.

\section{§3. Dual mappings}

In this section, we define the dual mapping of a given immersion and discuss 
its elementary properties. We assume the nondegeneracy throughout the section.

Let $\mathbf{R}_{n+2}$ denote the dual vector space of $\mathbf{R}^{n+2}$ and $\eta^{*}$ the radial vector field of $\mathbf{R}_{n+2}$. We define two mappings $v$ and $w$ from $M$ into $\mathbf{R}_{n+2}$ by associating to each point $x$ two linear functions $v(x)$ and $w(x)$ on $T_{f(x)} \mathbf{R}^{n+2}$, which is identified with the vector space $\mathbf{R}^{n+2}$, as follows:

$v(x)\left(\xi_{f(x)}\right)=1, \quad v(x)\left(\eta_{f(x)}\right)=0, \quad$ and $\quad v(x)\left(f_{*} X\right)=0 \quad$ for all $X \in T_{x} M$; $w(x)\left(\xi_{f(x)}\right)=0, \quad w(x)\left(\eta_{f(x)}\right)=1$, and $w(x)\left(f_{*} X\right)=0$ for all $X \in T_{x} M$.

Lemma 3.1. The derivatives of the mappings $v$ and $w$ are given as follows:

$$
\begin{array}{ll}
\left(D_{X} v\right)(\xi)=-\tau(X) & \left(D_{X} w\right)(\xi)=-\rho(X) \\
\left(D_{X} v\right)(\eta)=0 & \left(D_{X} w\right)(\eta)=0 \\
\left(D_{X} v\right)\left(f_{*} Y\right)=-h(X, Y) & \left(D_{X} w\right)\left(f_{*} Y\right)=-T(X, Y) .
\end{array}
$$

The nondegeneracy of $h$ implies that the mapping $v$ defines an immersion, because from the assumption $v_{*} Y=D_{Y} v=0$ follows $Y=0$ by the identity $\left(D_{X} v\right)\left(f_{*} Y\right)=-h(X, Y)$. Since $v\left(f_{*} X\right)=0$ and since $D_{Y} v$ is nonzero, the vector field $\eta^{*}$ is transversal to the mapping $v$. Since $w(\eta)=1$ and $D_{X} v(\eta)=0$, the vector field $w$ is also transversal to the mapping $v$. Because of the definition two vector fields $v$ and $w$ are linearly independent. So, the mapping $v$ defines a centroaffine immersion of $M$. The pair $\{v, w\}$ is called the dual mapping of $\{f, \xi\}$. The following set of equations

$$
\begin{aligned}
& D_{X} \eta^{*}=v_{*} X \\
& D_{X} v_{*} Y=T^{*}(X, Y) \eta^{*}+v_{*}\left(\nabla_{X}^{*} Y\right)+h^{*}(X, Y) w \\
& D_{X} w=\rho^{*}(X) \eta^{*}-v_{*}\left(S^{*} X\right)+\tau^{*}(X) w
\end{aligned}
$$

defines the objects $\nabla^{*}, h^{*}, T^{*}, S^{*}, \rho^{*}$, and $\tau^{*}$ for the dual mapping $v$.

LEMMA 3.2 .

$$
\begin{aligned}
& T^{*}(X, Y)=-h(S X, Y)+\tau(X) \tau(Y)-\left(\nabla_{X}^{*} \tau\right)(Y) \\
& h^{*}(X, Y)=h(X, Y) \\
& Z(h(X, Y))=h\left(\nabla_{Z} X, Y\right)+h\left(X, \nabla_{X}^{*} Y\right)+\tau(Y) h(X, Z) .
\end{aligned}
$$

Proof. These formulas are obtained by differentiating the three equations on the left hand side of Lemma 3.1. For example,

$$
\begin{aligned}
0 & =X\left\{\left(v_{*}(Y)\right)(\xi)+\tau(Y)\right\} \\
& =D_{X}\left(v_{*} Y\right)(\xi)+\left(v_{*} Y\right)\left(D_{X} \xi\right)+\left(\nabla_{X}^{*} \tau\right)(Y)+\tau\left(\nabla_{X}^{*} Y\right)
\end{aligned}
$$




$$
\begin{aligned}
= & \left(T^{*}(X, Y) \eta^{*}+v_{*}\left(\nabla_{X}^{*} Y\right)+h^{*}(X, Y) w\right)(\xi) \\
& +\left(v_{*} Y\right)\left(\rho(X) \eta-f_{*} S X+\tau(X) \xi\right)+\left(\nabla_{X}^{*} \tau\right)(Y)+\tau\left(\nabla_{X}^{*} Y\right) \\
= & \left\{T^{*}(X, Y)-\tau\left(\nabla_{X}^{*} Y\right)\right\}+\{h(S X, Y)-\tau(X) \tau(Y)\} \\
& +\left(\nabla_{X}^{*} \tau\right)(Y)+\tau\left(\nabla_{X}^{*} Y\right)
\end{aligned}
$$

shows the first formula. The other two are derived likewise.

Similar computation shows

LEMMA 3.3.

$$
\begin{aligned}
& \rho^{*}(X)=-\rho(X)-\tau\left(S^{*} X\right) \\
& \tau^{*}(X)=0 \\
& h\left(S^{*} X, Y\right)=-T(X, Y) .
\end{aligned}
$$

Recall the definition of $\mathscr{T}$ and define $\mathscr{T}^{*}$ by

$$
\mathscr{T}^{*}(X, Y)=T^{*}(X, Y)+h^{*}\left(S^{*} X, Y\right) .
$$

PROPOSITION 3.4

(1) $\mathscr{T}(X, Y)+\mathscr{T}^{*}(X, Y)=\tau(X) \tau(Y)-\left(\nabla_{X}^{*} \tau\right)(Y)$.

(2) $C^{*}(X, Y, Z)+C(X, Y, Z)=\tau(X) h(Y, Z)+\tau(Y) h(Z, X)+\tau(Z) h(X, Y)$.

Proof. The identity (1) follows from Lemma 3.2 and Lemma 3.3. We prove (2). By definition, we have

$$
C(X, Y, Z)=X(h(Y, Z))-h\left(\nabla_{X} Y, Z\right)-h\left(Y, \nabla_{X} Z\right)+\tau(X) h(Y, Z) .
$$

Since $h^{*}=h$ and $\tau^{*}=0$, we obtain

$$
C^{*}(X, Y, Z)=X(h(Y, Z))-h\left(\nabla_{X}^{*} Y, Z\right)-h\left(Y, \nabla_{X}^{*} Z\right) .
$$

Hence the sum of these equations gives the formula in view of the third identity of Lemma 3.2.

We remark here that when $\tau=0$ the formulas take simple forms; in particular, two connections $\nabla$ and $\nabla^{*}$ are conjugate to each other:

$$
X(h(Y, Z))=h\left(\nabla_{X} Y, Z\right)+h\left(Y, \nabla_{X}^{*} Z\right) .
$$

This is a well-known relation in affine hypersurface theory (see [DNV]).

Let us next consider the dual of the dual: denote by $(p, q)$ the dual of $(v, w)$. It is determined by 


$$
\begin{aligned}
& p(w)=1, \quad p(v)=0, \quad \text { and } \quad p\left(v_{*} X\right)=0 \\
& q(w)=0, \quad q(v)=1, \quad \text { and } q\left(v_{*} X\right)=0 \text {. }
\end{aligned}
$$

If we put $p_{x}=a \eta_{f(x)}+f_{* x}(V)+b \xi_{f(x)}$, then $a=p(w)=1, b=p(v)=0$, and $p\left(v_{*} X\right)=-h(X, V)-b \tau(X)=-h(X, V)$; since $h$ is nondegenerate, $V=0$. Thus $p_{x}=\eta_{f(x)} \equiv f(x)$. Similarly, we see $q_{x}=\xi_{x}+f_{* x} V$, where $V$ is defined by $h(X, V)+\tau(X)=0$. Therefore, the dual mapping of the dual is the same as the original immersion while the transversal vector field changes a little depending on $\tau$

Let $F$ be an immersion of $M$ into the projective space $\mathbf{P}^{n+1}$ and $f$ a local lift of $F:[f]=F$. The dual mapping $v$ is associated with a transversal vector field $\xi$. The dual mapping $v^{\prime}$ associated with another vector field $\xi^{\prime}=\left(\xi+a \eta+f_{*} U\right) / \lambda$ differs from $v$ by $v^{\prime}=\lambda v$. Hence, $[v]=\left[v^{\prime}\right]$ as mappings into the dual projective space $\mathbf{P}_{n+1}$. Let $g$ be another choice of local lift of $F$; then, $g=\phi f$ for a nonzero scalar function $\phi$. In this case, the dual mapping $v_{g}$ for $\{g, \xi\}$ is equal to $v$. So, we can define the dual immersion $F^{*}$ of $F$ by $F^{*}=[v]$. The discussion in the previous paragraph says that $\left(F^{*}\right)^{*}=F$.

We say that the pair $\{f, \xi\}$ is affinely selfdual if $f=A v$ for a linear isomorphism $A$ of $\mathbf{R}_{n+2}$ with $\mathbf{R}^{n+2}$. We say that the immersion $F=[f]$ is selfdual if $F=A F^{*}$ for a projective linear isomorphism $A$ of $\mathbf{P}_{n+1}$ with $\mathbf{P}^{n+1}$.

For both cases, denoting by $\langle$, $\rangle$ the dual pairing of $\mathbf{R}^{n+2}$ and $\mathbf{R}_{n+2}$, we have

$$
\left\langle f, A^{-1} f\right\rangle=\langle f, v\rangle=0 .
$$

This proves

PRoposition 3.5. The image of an affinely selfdual (nondegenerate) centroaffine immersion lies in a quadratic cone, that is, a cone over a quadratic hypersurface in an affine hyperplane not passing through the origin. The image of a selfdual (nondegenerate) projective immersion is part of a nondegenerate quadratic hypersurface.

\section{§4. Projective flatness and umbilicity}

Two torsion-free affine connections $\nabla$ and $\nabla^{\prime}$ are said to be projectively equivalent if there is a 1 -form $\sigma$ such that

$$
\nabla_{X}^{\prime} Y=\nabla_{X} Y+\sigma(X) Y+\sigma(Y) X .
$$

If $\sigma$ is closed, we say that $\nabla$ and $\nabla^{\prime}$ are projectively equivalent in a stronger sense. 
The connection $\nabla$ is said to be projectively flat if it is a projectively equivalent to a flat affine connection.

Let us recall the definition of the projective curvature tensor of a connection $\nabla$ ([E, p. 97]). Put

$$
W_{1}(X, Y) Z=R(X, Y) Z-\{\gamma(Y, Z) X-\gamma(X, Z) Y\},
$$

where $\gamma(X, Y)=\operatorname{Ric}(X, Y) /(n-1)$ and

$$
W_{2}(X, Y) Z=\frac{2}{n^{2}-1}(A(Y, Z) Y-A(X, Z) Y)+\frac{2}{n+1} A(X, Y) Z,
$$

where

$$
A(X, Y)=\frac{1}{2}(\operatorname{Ric}(X, Y)-\operatorname{Ric}(Y, X))
$$

Then the projective curvature tensor $W$ is defined by

$$
W(X, Y) Z=W_{1}(X, Y) Z+W_{2}(X, Y) Z .
$$

If $\nabla$ has symmetric Ricci tensor, $W_{2}=0$ and hence $W=W_{1}$.

If two affine connections $\nabla$ and $\nabla^{\prime}$ are projectively equivalent, they have the same $W$. It is known that $\nabla$ is projectively flat if its projective curvature tensor $W$ is identically zero when $n \geq 3$.

Denote by $S^{o}$ the traceless part of $S: S^{o}=S-(\operatorname{tr} S / n) I$. Then the identities (1.3) and (1.9) show

$$
\begin{aligned}
W_{1}(X, Y) Z & =h(Y, Z) S^{o} X-h(X, Z) S^{o} Y \\
+ & \frac{1}{n-1}\left\{h\left(S^{o} Y, Z\right) X-h\left(S^{o} X, Z\right) Y\right\}, \\
W_{2}(X, Y) Z & =\frac{1}{n^{2}-1}\left[\left\{h\left(S^{o} Z, Y\right)-h\left(S^{o} Y, Z\right)\right\} X\right. \\
& \left.-\left\{h\left(S^{o} Z, X\right)-h\left(S^{o} X, Z\right)\right\} Y\right] \\
+ & \frac{1}{n+1}\left\{h\left(S^{o} Y, X\right)-h\left(S^{o} X, Y\right)\right\} Z .
\end{aligned}
$$

We prove

THEOREM 4.1. Let $n \geq 3$. The connection $\nabla$ is projectively flat if and only if either (1) $h=0$, or (2) rank $h=1$ and $S^{o}=\nu \cdot I$ on ker $h$, or (3) $S^{o}=0$. 
Proof. First, we show the if-part. When (1) or (3) occurs, $W$ vanishes trivially. We assume the case (2). Let $Y \in \operatorname{ker} h$. Then

$$
\begin{gathered}
W_{1}(X, Y) Z=-\nu h(X, Z) Y-\frac{1}{n-1} h\left(S^{o} X, Z\right) Y \\
W_{2}(X, Y) Z=-\frac{1}{n^{2}-1}\left\{h\left(S^{o} Z, X\right)-h\left(S^{o} X, Z\right)\right\} Y .
\end{gathered}
$$

If $X \subset$ ker $h$ or $Z \in \operatorname{ker} h$, then $W=W_{1}+W_{2}=0$. Let $X=Z \notin$ ker $h$; then $W_{2}$ $=0$. Write $S^{o} X \equiv \lambda X \bmod \operatorname{ker} h$; then, since $\operatorname{tr} S^{o}=\lambda+(n-1) \nu=0$, we have $W_{1}=0$. Hence $W=0$.

Second, we prove the converse statement. Assume $W=0$ and rank $h=1$. Let $\left\{X_{1}, \ldots, X_{n}\right\}$ be a basis such that $h\left(X_{1}, X_{1}\right)= \pm 1$ and $\left\{X_{2}, \ldots, X_{n}\right\}$ generates ker $h$. When $Z=X_{1}$ and $X \neq Y \in\left\{X_{2}, \ldots, X_{n}\right\}$, possible because $n \geq 3$,

$$
\begin{aligned}
& W_{1}(X, Y) Z=\frac{1}{n-1}\left\{h\left(S^{o} Y, X_{1}\right) X-h\left(S^{o} X, X_{1}\right) Y\right\}, \\
& W_{2}(X, Y) Z=\frac{1}{n^{2}-1}\left\{h\left(S^{o} X, X_{1}\right) Y-h\left(S^{o} Y, X_{1}\right) X\right\} .
\end{aligned}
$$

Hence, $W=0$ implies $h\left(S^{o} X, X_{1}\right) Y=h\left(S^{o} Y, X_{1}\right) X$ and $h\left(S^{o} X, X_{1}\right)=0$ because $X$ and $Y$ are linearly independent. Namely, we see $S^{o}$ (ker $\left.h\right) \subset$ ker $h$. When $X=Z=X_{1}$ and $Y \in \operatorname{ker} h$ (accordingly, $S^{\circ} Y \in \operatorname{ker} h$ ), $W_{2}(X, Y) Z=0$ and

$$
W_{1}(X, Y) Z=-h\left(X_{1}, X_{1}\right) S^{o} Y-\frac{1}{n-1} h\left(S^{o} X_{1}, X_{1}\right) Y .
$$

Therefore, $S^{o} Y=\nu Y$ where $\nu=-h\left(S^{o} X_{1}, X_{1}\right) /(n-1) h\left(X_{1}, X_{1}\right)$, which shows the case (2).

Assume next rank $h \geq 2$ and $W=0$; we see $S^{0}=0$. Let $\left\{X_{1}, \ldots, X_{r}, X_{r+1}, \ldots\right.$, $\left.X_{n}\right\}$ be a basis such that $\left\{X_{r+1}, \ldots, X_{n}\right\}$ generates ker $h$ and $h\left(X_{i}, X_{j}\right)= \pm \delta_{i j}$ for $1 \leq i, j \leq r$. When $r \geq 3$ and $X \neq Y \neq Z \neq X$ are in $\left\{X_{1}, \ldots, X_{r}\right\}$,

$$
\begin{aligned}
W_{1}(X, Y) Z= & \frac{1}{n-1}\left\{h\left(S^{o} Y, Z\right) X-h\left(S^{o} X, Z\right) Y\right\} \\
W_{2}(X, Y) Z= & \frac{1}{n^{2}-1}\left\{h\left(S^{o} Z, Y\right)-h\left(S^{o} Y, Z\right)\right\} X \\
& -\frac{1}{n^{2}-1}\left\{h\left(S^{o} Z, X\right)-h\left(S^{o} X, Z\right)\right\} Y \\
& +\frac{1}{n+1}\left\{h\left(S^{o} Y, X\right)-h\left(S^{o} X, Y\right)\right\} Z
\end{aligned}
$$


Hence, when $W=0$, the coefficient of $Z$ says $h\left(S^{o} Y, X\right)=h\left(S^{o} X, Y\right)$ and, so, $W_{2}=0$; then the coefficient of $X$ in $W_{1}$ implies $h\left(S^{o} Y, Z\right)=0$.

When $Y \neq Z \in\left\{X_{1}, \ldots, X_{r}\right\}$ and $X \in\left\{X_{r+1}, \ldots, X_{n}\right\}$, we have

$$
\begin{aligned}
W_{1}(X, Y) Z= & \frac{1}{n-1}\left\{h\left(S^{o} Y, Z\right) X-h\left(S^{o} X, Z\right) Y\right\} \\
W_{2}(X, Y) Z= & \frac{1}{n^{2}-1}\left\{h\left(S^{o} Z, Y\right)-h\left(S^{o} Y, Z\right)\right\} X \\
& +\frac{1}{n^{2}-1} h\left(S^{o} X, Z\right) Y-\frac{1}{n+1} h\left(S^{o} X, Y\right) Z .
\end{aligned}
$$

The coefficient of $Z$ shows $h\left(S^{o} X, Y\right)=0$, i.e., $S^{o}$ ker $h \subset$ ker $h$. Then the coefficient of $X$ vanishes:

$$
\frac{1}{n-1} h\left(S^{o} Y, Z\right)+\frac{1}{n^{2}-1}\left\{h\left(S^{o} Z, Y\right)-h\left(S^{o} Y, Z\right)\right\}=0,
$$

from which it is easy to see $h\left(S^{o} Y, Z\right)=0$ also in this case. Hence we have seen that there exist scalars $\nu_{i}$ such that

$$
S^{o} X_{i} \equiv \nu_{i} X_{i} \quad \bmod \operatorname{ker} h, 1 \leq i \leq r .
$$

Now let both $Y=Z$ and $X$ belong to $\left\{X_{1}, \ldots, X_{r}\right\}$. Then, $W_{2}=0$ by (4.3) and

$$
W_{1}(X, Y) Z=h(Y, Y) S^{o} X+\frac{1}{n-1} h\left(S^{o} Y, Y\right) X .
$$

Hence, $\nu_{i}+\nu_{j} /(n-1)=0$ for $i \neq j$. Since $n \geq 3$, it is easy to see $\nu_{i}=0$. Then, $W_{1}=0$ implies now $S^{o} X=0$, i.e., $S^{o}=0$ on $\left\{X_{1}, \ldots, X_{r}\right\}$.

Consider finally the case where $Y=Z \in\left\{X_{1}, \ldots, X_{n}\right\}$ and $X \in\left\{X_{r+1}, \ldots, X_{n}\right\}$. We have $W_{2}=0$ and $W_{1}(X, Y) Z=h(Y, Y) S^{0} X$. Hence $S^{0}=0$ also on $\left\{X_{r+1}, \ldots\right.$, $X_{n}$ \}. This ends the proof.

Remark that the proof is the same as that of Theorem 5 of [NP3] when Ricci curvature is symmetric.

We say the immersion $f$ is umbilical relative to $\xi$ if $S^{o}=0$, i.e., $S=\nu I$ for some scalar function $\nu$.

Lemma 4.2. Assume $S=\nu I$ and $n \geq 2$. Then

(1) $d \tau=0$.

(2) $d \nu-\nu \tau+\rho=0$.

(3) $\alpha(\xi+\nu \eta)$ is a constant vector where $\alpha$ is defined (locally) by $\tau=-d \log \alpha$. 
Proof. (1) follows from the identity (1.8). For (3), it is enough to check

$$
\begin{aligned}
D_{X}(\xi+\nu \eta) & =(\rho(X) \eta-S X+\tau(X) \xi)+X(\nu) \eta+\nu X \\
& =\tau(X)(\xi+\nu \eta) .
\end{aligned}
$$

The assumption $S=\nu I$ implies $\nabla_{X} S=(X \nu) \cdot I$. Then, by (1.6),

$$
\{X(\nu)-\nu \tau(X)+\rho(X)\} Y=\{Y(\nu)-\nu \tau(Y)+\rho(Y)\} X .
$$

If $n \geq 2$, (2) follows.

THEOREM 4.3. Assume the immersion $f$ is umbilical. Then each 2-dimensional linear subspace spanned by $\eta_{x}$ and $\xi_{x}$ contains a fixed line through the origin; in other words, each projective line through $[f(x)]$ in $\mathbf{P}^{n+1}$ in the direction of $\left[\xi_{x}\right]$ passes through a fixed point.

Proof. Put $y=f(x)+\lambda \xi+\mu \eta$. Then we get

$$
D_{X} y=(\lambda \rho(X)+X \mu) \eta+(X-\lambda S X+\mu X)+(X \lambda+\lambda \tau(X)) \xi .
$$

Letting $\mu=\lambda \nu-1$ and using (2) of Lemma 4.2, we check

$$
D_{X} y=(X \lambda+\lambda \tau(X))(\xi+\nu \eta) .
$$

Put $\mathbf{a}=\alpha(\xi+\nu \eta)$ and $\omega=\alpha^{-1}(d \lambda+\lambda \tau)$. Then we obtain

$$
D_{X} y=\omega(X) \cdot \mathbf{a},
$$

which proves the conclusion.

Remark. Our method in this paper makes it possible to recapture Proposition 8 in [NP3] without the assumption of equiprojectivity.

\section{§5. Uniqueness theorems}

In this section we are going to establish a number of uniqueness theorems for centroaffine immersions $M \rightarrow \mathbf{R}^{n+2}-\{0\}$ and for immersions $M \rightarrow \mathbf{P}^{n+1}$.

Consider two immersions $f^{i}: M \rightarrow \mathbf{R}^{n+2}-\{0\}, i=1,2$, with transversal vector fields $\xi^{i}$. We have two sets of invariants $\left(\nabla^{i}, h^{i}, T^{i}, S^{i}, \rho^{i}, \tau^{i}\right)$. We say that $f^{1}$ and $f^{2}$ are affinely (resp. projectively) equivalent if $f^{1}=A f^{2}$ (resp. if $\left.\left[f^{1}\right]=\left[A f^{2}\right]\right)$ for a general linear transformation $A$ in $G L(n+2, \mathbf{R})$.

A preliminary uniqueness theorem is stated as follows. 
Lemma 5.1. Assume $\nabla^{1}=\nabla^{2}, h^{1}=h^{2}, T^{1}=T^{2}, S^{1}=S^{2}, \rho^{1}=\rho^{2}$, and $\tau^{1}=\tau^{2}$. Then $f^{1}$ and $f^{2}$ are affinely equivalent.

We follow the patterns in $[\mathrm{D}]$ and $[\mathrm{O}]$ to prove the following.

Lemma 5.2 Assume $\nabla^{1}=\nabla^{2}=: \nabla, h^{1}=h^{2}=: h$, and $T^{1}=T^{2}=:$. If rank $h \geq 2$, then $f^{1}$ and $f^{2}$ are affinely equivalent.

Proof. From the equation (1.5),

$$
\begin{aligned}
\left(\nabla_{X} h\right)(Y, Z)-\left(\nabla_{Y} h\right)(X, Z) & =\tau^{1}(Y) h(X, Z)-\tau^{1}(X) h(Y, Z) \\
& =\tau^{2}(Y) h(X, Z)-\tau^{2}(X) h(Y, Z) .
\end{aligned}
$$

Hence, for $\tau:=\tau^{1}-\tau^{2}$, we get

$$
\tau(Y) h(X, Z)=\tau(X) h(Y, Z) .
$$

Then, the assumption rank $h \geq 2$ implies $\tau=0$ by Lemma 1.2. Similarly, for $\rho:=\rho^{1}-\rho^{2}$, the equation (1.4) shows

$$
\rho(Y) h(X, Z)=\rho(X) h(Y, Z)
$$

and we get $\rho=0$. Lastly, for $S:=S^{1}-S^{2}$, the equation (1.3) gives

$$
h(Y, Z) S X=h(X, Z) S Y
$$

which implies $S=0$. Hence the equivalence follows from Lemma 5.1.

We shall further prove

Lemma 5.3. Assume $\nabla^{1}=\nabla^{2}, h^{1}=\lambda h^{2}$, and $T^{1}=T^{2}-a h^{2}$, where $a$ and $\lambda$ are scalar functions and $\lambda$ is nonzero. If rank $h^{1} \geq 2$, then $f^{1}$ and $f^{2}$ are affinely equivalent.

Proof. Put $\xi^{2^{\prime}}=\left(\xi^{2}-a \eta\right) / \lambda$. Then relative to $\xi^{2^{\prime}}$ we see

$$
h^{2^{\prime}}=\lambda h^{2}, \quad T^{2^{\prime}}=T^{2}-a h^{2}, \nabla^{2^{\prime}}=\nabla^{2} ;
$$

hence, we can apply Lemma 5.2.

The assumption of Lemma 5.2 can be modified to yield projective equivalence:

LEMma 5.4. Assume

$$
\nabla_{X}^{1} Y=\nabla_{X}^{2} Y+\sigma(Y) X+\sigma(X) Y
$$




$$
\begin{aligned}
& T^{1}(X, Y)=T^{2}(X, Y)+\left(\nabla_{X} \sigma\right)(Y)-\sigma(X) \sigma(Y) \\
& h^{1}(X, Y)=\lambda h^{2}(X, Y)
\end{aligned}
$$

where $\lambda$ is a nonzero scalar function and $\sigma$ is a closed 1 -form. Then $f^{1}$ and $f^{2}$ are projectively equivalent.

Proof. Let $\sigma=d \log \mu$ locally and consider the immersion $g=\mu f^{2}$ with the same transversal $\xi^{2}$. Then

$$
\begin{aligned}
& \nabla_{X}^{g} Y=\nabla_{X}^{2} Y+\sigma(Y) X+\sigma(X) Y \\
& T^{g}(X, Y)=T^{2}(X, Y)+\left(\nabla_{X} \sigma\right)(Y)-\sigma(X) \sigma(Y) \\
& h^{g}(X, Y)=\mu h^{2}(X, Y) .
\end{aligned}
$$

Hence $\nabla^{1}=\nabla^{g}, T^{1}=T^{g}$, and $h^{1}=(\lambda / \mu) h^{g}$. Apply Lemma 5.3 to $f^{1}$ and $g$ to obtain the result.

Now we can drop the condition on $T$. We prove

Proposition 5.5. Let $n \geq 3$. Assume $\nabla^{1}=\nabla^{2}=: \nabla$ and $h^{1}=h^{2}=: h$. If rank $h \geq 2$, then $f^{1}$ and $f^{2}$ are affinely equivalent.

Proof. Let us recall that the projective curvature tensor $W$ is the sum of two tensors $W_{1}$ and $W_{2}$ which have expressions given in (4.1) and (4.2). The right-hand side of $W$ has two expressions, one using $S^{10}$ and the other using $S^{20}$. Hence, for $S^{o}=S-(\operatorname{tr} S / n) I$ where $S:=S^{1}-S^{2}$, we see

$$
\begin{gathered}
h(Y, Z) S^{o} X-h(X, Z) S^{o} Y+\frac{1}{n-1}\left\{h\left(S^{o} Y, Z\right) X-h\left(S^{o} X, Z\right) Y\right\} \\
+\frac{1}{n^{2}-1}\left[\left\{h\left(S^{o} Z, Y\right)-h\left(S^{o} Y, Z\right)\right\} X-\left\{h\left(S^{o} Z, X\right)-h\left(S^{o} X, Z\right)\right\} Y\right] \\
+\frac{1}{n+1}\left\{h\left(S^{o} Y, X\right)-h\left(S^{o} X, Y\right)\right\} Z=0 .
\end{gathered}
$$

Since rank $h \geq 2$, the argument in the proof of Proposition 4.1 works and we have $S^{o}=0$, i. e., $S=s I$ for a scalar $s$. Now, the equation (1.3) implies, for $T:=$ $T^{1}-T^{2}$

$$
\operatorname{sh}(Y, Z) X-\operatorname{sh}(X, Z) Y-T(Y, Z) X+T(X, Z) Y=0 .
$$

Hence, $T(Y, Z)=\operatorname{sh}(Y, Z)$. Take $\xi^{2^{\prime}}=\xi^{2}-s \eta$; then, $T^{2^{\prime}}=T^{2}+s h$ by (1.12) and $S^{2^{\prime}}=S^{2}+s I$ by (1.16). This means, in particular, $T^{1}-T^{2^{\prime}}=0$ and we can 
apply Lemma 5.2 .

THEOREM 5.6. Let $n \geq 3$. Given two centroaffine immersions $f^{1}$ and $f^{2}, M \rightarrow$ $\mathbf{R}^{n+2}-\{0\}$, assume that the induced connections $\nabla^{1}$ and $\nabla^{2}$ coincide and that $h^{1}$ and $h^{2}$ are conformal and of rank $\geq 2$. Then both immersions are affinely equivalent.

Proof. By scaling $\xi^{2}$ by an appropriate nonzero factor, we can reduce the case to that of Proposition 5.5 in view of (1.11) and (1.13).

THEOREM 5.7. Let $n \geq 3$. Assume $\nabla^{1}$ is projectively equivalent in a stronger sense to $\nabla^{2}$ and $h^{1}$ is conformally equivalent to $h^{2}$. If rank $h^{1} \geq 2$, then $f^{1}$ is projectively equivalent to $f^{2}$.

Proof. By scaling $f^{2}$ by an appropriate scalar, we can reduce the case to that in the previous proposition.

Two theorems above can be further generalized. We consider a pair $(\nabla, h)$ which arises from an immersion $f: M \rightarrow \mathbf{R}^{n+2}-\{0\}$ together with a transversal vector field $\xi$. In the set of all such pairs $(\nabla, h)$ associated to all immersions $M \rightarrow \mathbf{R}^{n+2}-\{0\}$, we define an equivalence relation: $(\nabla, h) \sim_{a}\left(\nabla^{\prime}, h^{\prime}\right)$ if there exist a vector field $U$ and a function $\lambda \neq 0$ on $M$ such that

$$
\nabla_{X}^{\prime} Y=\nabla_{X} Y-h(X, Y) U \text { and } h^{\prime}=\lambda h .
$$

It is easily checked that, given $f: M \rightarrow \mathbf{R}^{n+2}-\{0\}$, we get an equivalence class $[(\nabla, h)]_{a}$ independently of the choice of $\xi$.

THEOREM 5.8. Let $n \geq 3$. Two immersions $f^{1}, f^{2}: M \rightarrow \mathbf{R}^{n+2}-\{0\}$ of rank $\geq 2$ are affinely equivalent if and only if the equivalence classes $\left[\left(\nabla^{1}, h^{1}\right)\right]_{a}$ and $\left[\left(\nabla^{2}, h^{2}\right)\right]_{a}$ for $f^{1}$ and $f^{2}$ coincide.

Proof. See the equations (1.11) and (1.13).

Given two immersions $F^{1}, F^{2}: M \rightarrow \mathbf{P}^{n+1}$, we shall say that $F^{1}$ and $F^{2}$ are projectively equivalent if there is a projective transformation $\tilde{A}$ of $\mathbf{P}^{n+1}$ such that $F^{2}$ $=\tilde{A} \cdot F^{1}$. In this case, any lift $f^{1}$ of $F^{1}$ and any lift $f^{2}$ of $F^{2}$ are projectively equivalent in the sense we defined in the beginning.

Given an immersion $F: M \rightarrow \mathbf{P}^{n+1}$, we consider a pair $(\nabla, h)$ which arises from the choice of a lift $f: M \rightarrow \mathbf{R}^{n+2}-\{0\}$ together with a transversal vector 
field $\xi$. In the set of all such pairs $(\nabla, h)$ associated to all immersions $M \rightarrow \mathbf{P}^{n+1}$, we define an equivalence relation: $(\nabla, h) \sim_{p}\left(\nabla^{\prime}, h^{\prime}\right)$ if there exist a closed 1 -form $\sigma$, a vector field $U$, and a function $\lambda \neq 0$ on $M$ such that

$$
\nabla_{X}^{\prime} Y=\nabla_{X} Y+\sigma(X) Y+\sigma(Y) X-h(X, Y) U \text { and } h^{\prime}=\lambda h .
$$

It is easily checked by (1.11) and (2.2) that, given $F: M \rightarrow \mathbf{P}^{n+1}$, we get an equivalence class $[(\nabla, h)]_{p}$ independently of the choice of $\{f, \xi\}$ representing $F$. We define the rank of $F$ as the rank of $h$. We can now state

Theorem 5.9. Let $n \geq 3$. Two immersions $F^{1}, F^{2}: M \rightarrow \mathbf{P}^{n+1}$ of rank $\geq 2$ are projectively equivalent if and only if the equivalence classes $\left[\left(\nabla^{1}, h^{1}\right)\right]_{p}$ and $\left[\left(\nabla^{2}, h^{2}\right)\right]_{p}$ for $F^{1}$ and $F^{2}$ coincide.

Remark. Formula (5.2) appears in [NP3], (44), as well as in [S].

\section{§6. Immersions with $\nabla h=0$}

In affine hypersurface theory a well-known theorem of Pick and Berwald can be formulated as follows. If a nondegenerate hypersurface has vanishing cubic form, then it lies in a quadric. In this section we shall obtain a result of this type for centroaffine immersions $M \rightarrow \mathbf{R}^{n+2}-\{0\}$.

Lemma 6.1. Assume rank $h \geq 2$ and $R(X, Y) h=0$. Then $d \tau=0$ and $\mathscr{T}=H h$, i.e., $h(S X, Y)+T(X, Y)=H h(X, Y)$ for some scalar function $H$.

Proof. By the assumption, we have

$$
h(R(X, Y) Y, Z)+h(Y, R(X, Y) Z)=-(R(X, Y) h)(Y, Z)=0 .
$$

Then from (1.3) we have

$$
\begin{aligned}
& h(Y, Y) h(S X, Z)-h(X, Y) h(S Y, Z)+T(X, Y) h(Y, Z) \\
(6.1)- & T(Y, Y) h(X, Z)+h(Y, Z) h(S X, Y)-h(X, Z) h(S Y, Y) \\
+ & T(X, Z) h(Y, Y)-T(Y, Z) h(X, Y)=0 .
\end{aligned}
$$

Let $\left\{X_{1}, \ldots, X_{r}, X_{r+1}, \ldots, X_{n}\right\}$ be a basis such that $\left\{X_{r+1}, \ldots, X_{n}\right\}$ generates ker $h$ and $h\left(X_{i}, X_{j}\right)=\varepsilon_{i} \delta_{i j}, \varepsilon_{i}= \pm 1$, for $1 \leq i, j \leq r$. Let $1 \leq j \leq r$ and $1 \leq i \leq n$ with $i \neq j$. By setting $X=X_{i}, Y=Z=X_{j}$, (6.1) implies

$$
h\left(S X_{i}, X_{j}\right)+T\left(X_{i}, X_{j}\right)=0 .
$$


Next, let $r+1 \leq j \leq r$ and $1 \leq i \leq n$ with $i \neq j$. Choose $k, 1 \leq k \leq r, k \neq i$ (which is possible by virtue of the assumption rank $h \geq 2$ ). By setting $X=X_{i}$, $Y=X_{k}$ and $Z=X_{j}$, (6.1) again implies (6.2). Thus (6.2) holds for all $i, j(i \neq j)$.

Now let $1 \leq i, j \leq n, i \neq j$. If we set $X=Z=X_{i}, Y=X_{j}$, then (6.1) leads to

$$
\begin{aligned}
& h\left(X_{j}, X_{j}\right)\left\{h\left(S X_{i}, X_{i}\right)+T\left(X_{i}, X_{i}\right)\right\} \\
& =h\left(X_{i}, X_{i}\right)\left\{h\left(S X_{j}, X_{j}\right)+T\left(X_{j}, X_{j}\right)\right\} \text { for } 1 \leq i, j \leq r \\
& h\left(S X_{i}, X_{i}\right)+T\left(X_{i}, X_{i}\right)=0 \text { for } r+1 \leq i, n .
\end{aligned}
$$

(6.2) and (6.3) together imply

$$
\varepsilon_{i} \mathscr{T}\left(X_{i}, X_{i}\right)=\varepsilon_{j} \mathscr{T}\left(X_{j}, X_{j}\right) \text { and } \mathscr{T}\left(X_{i}, X_{j}\right)=0(i \neq j) .
$$

Hence, there exists a function $H$ such that $\mathscr{T}\left(X_{i}, X_{j}\right)=H h\left(X_{i}, X_{j}\right)$ for any $i, j$. This proves the conclusion.

Lemma 6.2. Assume rank $h \geq 2, \nabla h=0$, and $n \geq 2$. Then

$$
d H+2 \rho=0 \text {. }
$$

Proof. Under $\nabla h=0,(1.5)$ becomes $\tau(X) h(Y, Z)=\tau(Y) h(X, Z)$; this impies $\tau=0$ by Lemma 1.2. Since $\nabla h=0$, we have $R(X, Y) h=0$ and

$$
h(S X, Y)+T(X, Y)=H h(X, Y)
$$

by Lemma 6.1. Differentiating this equation and using the assumption $\nabla h=0$, we get

$$
h\left(\left(\nabla_{Z} S\right) X, Y\right)+\left(\nabla_{Z} T\right)(X, Y)=(Z H) h(X, Y) .
$$

On the other hand, (1.4) and (1.6) imply

$$
\begin{aligned}
& h\left(\left(\nabla_{Z} S\right) X, Y\right)+\rho(Z) h(X, Y)=h\left(\left(\nabla_{X} S\right) Z, Y\right)+\rho(X) h(Z, Y) \\
& \left(\nabla_{Z} T\right)(X, Y)+\rho(Z) h(X, Y)=\left(\nabla_{X} T\right)(Y, Z)+\rho(X) h(Y, Z) .
\end{aligned}
$$

Hence, from the last three equations, we get

$$
Z(H) h(X, Y)+2 \rho(Z) h(X, Y)=X(H) h(Y, Z)+2 \rho(X) h(Y, Z) .
$$

This identity implies the result by Lemma 1.2.

We define for each $x \in M$ a quadratic cone through $x$ by the following equation:

$$
Q_{x}=\left\{\alpha(x+U+\mu \xi) \mid h(U, U)+H \mu^{2}-2 \mu=0, \quad \alpha \in \mathbf{R}^{*}\right\} .
$$


This is an analogue of Lie's quadrics ([B], p. 228-9). We now prove

THEOREM 6.3. Assume the immersion $M \rightarrow \mathbf{R}^{n+2}-\{0\}$ satisfies that rank $h \geq$ $2, \nabla h=0$, and $n \geq 2$. Then the image lies on a quadratic cone.

Proof. Fix a point $y \in \mathbf{R}^{n+2}-\{0\}$. For each $x \in M, y$ can be written as

$$
y=\alpha(x+U+\mu \xi) .
$$

Suppose $y \in Q_{x_{0}}$. Then, if we can show $y \in Q_{x}$ for every $x$, the proof is complete. To see this we compute $D_{X} y$ by using the fundamental equations. The result is

$$
\begin{aligned}
D_{X} y= & \alpha\{X(\log \alpha)+T(X, U)+\mu \rho(X)\} \eta \\
& +\alpha\left(X(\log \alpha) U+X+\nabla_{X} U-\mu S X\right) \\
& +\{X(\log \alpha) \mu+h(X, U)+X \mu\} \xi .
\end{aligned}
$$

Note here that $\tau=0$. On the other hand, since $y$ is fixed, we have $D_{X} y=0$; so,

$$
\begin{aligned}
T(X, U) & =-X(\log \alpha)-\mu \rho(X) \\
\nabla_{X} U & =\mu S X-X-X(\log \alpha) U \\
h(X, U) & =-\mu X(\log \alpha)-X \mu .
\end{aligned}
$$

Using these equations, we get

$$
\begin{aligned}
h\left(\nabla_{X} U, U\right) Z= & h(\mu S X-X-X(\log \alpha) U, U) \\
= & \mu h(S X, U)-h(X, U)-X(\log \alpha) h(U, U) \\
= & \mu(H h(X, U)-T(X, U))-h(X, U)-X(\log \alpha) h(U, U) \\
= & \mu\{X(\log \alpha)+\mu \rho(X) \\
& \quad+(H \mu-1)\{-\mu X(\log \alpha)-X \mu\}-X(\log \alpha) h(U, U)
\end{aligned}
$$

and, hence,

$$
\begin{aligned}
& X\left(h(U, U)+H \mu^{2}-2 \mu\right) \\
& \quad=2 h\left(\nabla_{X} U, U\right)+X H \cdot \mu^{2}+2 H \mu X \mu-2 X \mu \\
& \quad=2 X(\log \alpha)\{\mu-h(U, U)-\mu(H \mu-1)\}+(2 \rho(X)+X H) \mu^{2} .
\end{aligned}
$$

Therefore, by Lemma 6.2,

$$
X\left(h(U, U)+H \mu^{2}-2 \mu\right)=-2 X(\log \alpha)\left(h(U, U)+H \mu^{2}-2 \mu\right) ;
$$

this implies that $\alpha^{2}\left(h(U, U)+H \mu^{2}-2 \mu\right)$ is constant. Since it is zero at $x=x_{0}$, we have $h(U, U)+H \mu^{2}-2 \mu=0$. 
Lemma 6.4. Assume the cubic form $C$ vanishes and rank $h \geq 2$. Then $d \tau=0$ and $R(X, Y) h=0$.

Proof. The assumption $C=0$ means $\left(\nabla_{X} h\right)(U, V)=-\tau(X) h(U, V)$. Hence,

$$
\begin{aligned}
\left(\nabla_{X} \nabla_{Y} h\right)(U, V) & =X\left\{\left(\nabla_{Y} h\right)(U, V)\right\}-\nabla_{Y} h\left(\nabla_{X} U, V\right)-\left(\nabla_{Y} h\right)\left(U, \nabla_{X} V\right) \\
& =X(-\tau(Y) h(U, V))+\tau(Y) h\left(\nabla_{X} U, V\right)+\tau(Y) h\left(U, \nabla_{X} V\right) \\
& =-X(\tau(Y)) h(U, V)-\tau(Y)\left\{\left(\nabla_{X} h\right)(U, V)\right\} \\
& =\{\tau(X) \tau(Y)-X(\tau(Y))\} h(U, V) .
\end{aligned}
$$

Similarly,

$$
\begin{gathered}
\left(\nabla_{Y} \nabla_{X} h\right)(U, V)=\{\tau(X) \tau(Y)-Y(\tau(X))\} h(U, V), \\
\left(\nabla_{[X, Y]} h\right)(U, V)=-\tau([X, Y]) h(U, V) .
\end{gathered}
$$

Therefore, we get

$$
\begin{aligned}
(R(X, Y) h)(U, V) & =\{Y(\tau(X))-X(\tau(Y))+\tau([X, Y])\} h(U, V) \\
& =-d \tau(X, Y) h(U, V) .
\end{aligned}
$$

Then the identity $(R(X, Y) h)(U, V)+h(R(X, Y) U, V)+h(U, R(X, Y) V)$ $=0$ implies

$$
\begin{aligned}
& \{h(X, S Y)-h(Y, S X)\} h(U, V) \\
& =h(Y, U) h(S X, V)-h(X, U) h(S Y, V)+T(X, U) h(Y, V)-T(Y, U) h(X, V) \\
& +h(Y, V) h(S X, U)-h(X, V) h(S Y, U)+T(X, V) h(Y, U)-T(Y, V) h(X, U) .
\end{aligned}
$$

Let $\left\{X_{1}, \ldots, X_{r}, X_{r+1}, \ldots, X_{n}\right\}$ be a basis as in Lemma 6.1. For any $1 \leq i \leq r$, $1 \leq j \leq n, i \neq j$, by letting $X=X_{j}$, and $Y=U=V=X_{i}$, we get

$$
h\left(X_{j}, S X_{i}\right)-h\left(X_{i}, S X_{j}\right)=2\left\{h\left(S X_{j}, X_{i}\right)+T\left(X_{j}, X_{i}\right)\right\} .
$$

Assuming further $1 \leq j \leq r$ and interchanging $i$ and $j$, we have

$$
h\left(X_{i}, S X_{j}\right)-h\left(X_{j}, S X_{i}\right)=2\left\{h\left(S X_{i}, X_{j}\right)+T\left(X_{i}, X_{j}\right)\right\} .
$$

Since $T$ is symmetric, the difference implies

$$
h\left(X_{j}, S X_{i}\right)-h\left(X_{i}, S X_{j}\right)=0
$$

for $1 \leq i, j \leq r$.

Now for $r+1 \leq i \leq n, 1 \leq j \leq n, i \neq j$, take $1 \leq k \leq r, k \neq j$. By letting $X=X_{j}, Y=X_{i}, U=V=X_{k}$, we obtain

$$
h\left(X_{j}, S X_{i}\right)-h\left(X_{i}, S X_{j}\right)=0 .
$$


This proves (6.5) generally; hence $d \tau=0$ by (1.8).

Corollary 6.5. Assume the cubic form $C$ vanishes and rank $h \geq 2$. Then the image lies on a quadratic cone.

Proof. If $C=0$, then $\tau(X) h(Y, Z)=\tau(Y) h(X, Z)$ by (1.5). Lemma $1.2 \mathrm{im}$ plies $\tau=0$ and, then, $\nabla h=0$. Theorem 6.3 proves the result.

COROLlary 6.6. Assume the immersion $M \rightarrow \mathbf{R}^{n+2}-\{0\}$ satisfies the condition $h \mid C$, namely

$$
C(X, Y, Z)=h(X, Y) h(Z, U)+h(Y, Z) h(X, U)+h(Z, X) h(Y, U) .
$$

Then the image lies on a quadratic cone.

Proof. Proposition 1.7 implies that we can assume $C=0$ by a rechoice of a transversal field $\xi$.

\section{§7. Immersions with $\nabla T=0$}

In this section we prove a result (Theorem 7.3) which is an analogue of Theorem 6.3.

Lemma 7.1. Assume rank $h \geq 2$. Then the condition $R(X, Y) T=0$ is equivalent to the condition $T(S X, Y)=k h(X, Y)$ for some scalar function $k$.

Proof. The condition $R(X, Y) T=0$ implies

$$
T(R(X, Y) U, V)+T(U, R(X, Y) V)=0 .
$$

Hence, by (1.3) and by the symmetry of $T$, we have

$$
\begin{aligned}
A:=h(Y, U) T & (S X, V)-h(X, U) T(S Y, V) \\
& +h(Y, V) T(S X, U)-h(X, V) T(S Y, U)=0 .
\end{aligned}
$$

Let $\left\{X_{1}, \ldots, X_{r}, X_{r+1}, \ldots, X_{n}\right\}$ be a basis as before. Suppose $j \neq k$ and at least one of them is in $\{r+1, \ldots, n\}$. Choose $1 \leq i \leq r, i \neq j, k$. By letting $X=X_{j}, Y=$ $U=X_{i}, V=X_{k}$, we obtain $T\left(S X_{j}, X_{k}\right)=0$. Suppose $j \neq k$ and both in $\{1, \ldots$ $r$. By letting $X=X_{j}, Y=U=V=X_{k}$, we obtain $T\left(S X_{j}, X_{k}\right)=0$.

Next, assuming $j \neq k$, let $X=V=X_{k}, Y=U=X_{j}$, we get 


$$
h\left(X_{j}, X_{j}\right) T\left(S X_{k}, X_{k}\right)-h\left(X_{k}, X_{k}\right) T\left(S X_{j}, X_{j}\right)=0 .
$$

Hence we see $T(S X, Y)=k h(X, Y)$. The converse can be seen by showing $A=0$ under this condition.

Lemma 7.2. Assume $\nabla T=0, T(S X, Y)=k h(X, Y)$, and rank $h \geq 2$. Then

$$
d \log k=2 \tau \text {. }
$$

Proof. By differentiating $T(S X, Y)=k h(X, Y)$, we get

$$
T\left(X,\left(\nabla_{Z} S\right) Y\right)=Z(k) h(X, Y)+k\left(\nabla_{Z} h\right)(X, Y) .
$$

By interchanging $Y$ and $Z$ and by taking the difference, we get

$$
\begin{aligned}
& T\left(X,\left(\nabla_{Z} S\right) Y-\left(\nabla_{Y} S\right) Z\right) \\
& \quad=Z(k) h(X, Y)-Y(k) h(X, Z)+k\left\{\left(\nabla_{Z} h\right)(X, Y)-\left(\nabla_{Y} h\right)(X, Z)\right\} .
\end{aligned}
$$

Then equations (1.5) and (1.6) imply

$$
\{Z k-2 k \tau(Z)\} h(X, Y)=\{Y k-2 k \tau(Y)\} h(X, Z) .
$$

This implies the formula by Lemma 1.2 .

THEOREM 7.3. Assume the immersion $M \rightarrow \mathbf{R}^{n+2}-\{0\}$ satisfies that rank $h \geq 2, \nabla T=0$, and $n \geq 2$. Then the image lies on a quadratic hypersurface or on an affine hyperplane.

Proof. By the assumption we have from Lemmas 7.1 and 7.2
(1) $\rho=0$,
(2) $T(X, S Y)=k h(X, Y)$
(3) $d \log k=2 \tau$.

The first one follows from (1.4). We define a quadratic form $q_{x}$ on each tangent space $T_{f(x)} \mathbf{R}^{n+2}$ as follows:
(4) $q_{x}(\eta, \eta)=1$
(5) $q_{x}\left(f_{*} X, \eta\right)=0$
(6) $q_{x}(\xi, \eta)=0$
(7) $q_{x}\left(\xi, f_{*} X\right)=0$
(8) $q_{x}(\xi, \xi)=-k$
(9) $q_{x}\left(f_{*} X, f_{*} Y\right)=-T(X, Y)$.

If we can see that $q$ is $D$-parallel, then the proof is complete because the equation (4) represents a quadratic hypersurface or an affine hyperplane. The fact $D_{X} q=0$ is seen by simple computation. For example,

$$
\begin{aligned}
& \left(D_{X} q\right)\left(f_{*} Y, f_{*} Z\right) \\
& \quad=-q\left(D_{X}\left(f_{*} Y, f_{*} Z\right)-q\left(f_{*} Y, D_{X} f_{*} Z\right)-X(T(Y, Z))\right. \text { by (9) }
\end{aligned}
$$




$$
\begin{aligned}
= & -q\left(T(X, Y) \eta+f_{*} \nabla_{X} Y+h(X, Y) \xi, f_{*} Z\right) \\
& -q\left(f_{*} Y, T(X, Z) \eta+f_{*} \nabla_{X} Z+h(X, Z) \xi\right) \\
& -\left\{\left(\nabla_{X} T\right)(Y, Z)+T\left(\nabla_{X} Y, Z\right)+T\left(Y, \nabla_{X} Z\right)\right\} \\
= & 0 \text { by (2), (5), (7) and (9). }
\end{aligned}
$$

Remark. If $T \equiv 0$ in Theorem 7.3 , then also $k \equiv 0$; hence the quadratic form $q$ is of rank 1 and the hypersurface $q=1$ is an affine hyperplane. See Proposition 1.3. In general, at a point where $k \neq 0$ and $h$ is nondegenerate, the quadratic form $T$ is nondegenerate; hence the quadratic hypersurface is also nondegenerate.

The meaning of Theorem 7.3 may become clearer if we start with a quadratic form $q$ and assume that a centroaffine immersion $f: M \rightarrow \mathbf{R}^{n+2}-\{0\}$ is contained in the quadratic hypersurface $q(\eta, \eta)=1$, i.e. (4). Then (5) holds. By choosing $\xi$ satisfying (6), (7) and (8), we can see that these conditions imply (9) and thus lead to $\nabla T=0$. Namely, $f(M)$ contained in the quadratic hypersurface (4) satisfies $\nabla T=0$.

\section{REFERENCES}

[B] W. Blaschke, Vorlesungen über Differentialgeometrie II, Affine Differentialgeometrie, Springer, Berlin, 1923.

[D] F. Dillen, Equivalence theorems in affine differential geometry, Geom. Dedicata, 32 (1988), 81-92.

[DNV] F. Dillen, K. Nomizu, and L. Vranken, Conjugate connections and Radon's theorem in affine differential geometry, Monatsh. Math., 109 (1990), 221-235.

[E] L. P. Eisenhart, Non-Riemannian Geometry, Amer. Math. Soc. Colloq. Publ., 8, 1927.

[NP1] K. Nomizu and U. Pinkall, On the geometry of affine immersions, Math, Z., 195 (1987), 165-178.

[NP2] K. Nomizu and U. Pinkall, Cubic form theorem for affine immersions, Results in Math., 13 (1988), 338-362.

[NP3] K. Nomizu and U. Pinkall, On the geometry of projective immersions, J. Math. Soc. Japan, 41 (1989), 607-623.

[NS] K. Nomizu and T. Sasaki, On the classification of projectively homogeneous surfaces, Results in Math., 20 (1991), 698-724.

[O] B. Opozda, Some equivalence theorems in affine hypersurface theory, preprint 1991.

[S] T. Sasaki, On the projective geometry of hypersurfaces, MPI-preprint 86-7, Max-Planck-Institut für Mathmatik.

[W] R. Walter, Centroaffine differential geometry: submanifolds of codimension 2, Results in Math., 13 (1988), 386-402. 
Katsumi Nomizu

Department of Mathematics

Brown University

Providence, RI 02912

USA

Takeshi Sasaki

Dapartment of Mathematics

Kobe University

Rokko, Kobe 657

Japan 